\title{
Study on Relieving Cerebral Injury of CPB Rats by Danhong Injection
}

\author{
Kun Zhang, Lishen Wang ${ }^{*}$, Shuwei Shen, Aiping Dong, Rong Wang \\ Department of Anesthesiology, Jingzhou Central Hospital,The Second Clinical Medical College, \\ Yangtze University, Jingzhou, Hubei 434020,China. \\ ${ }^{*}$ Corresponding author: Dr. Lishen Wang \\ E-mail:6939904@qq.com
}

Keywords: Danhong injection; CPB; rat; cerebrum; protection effect

Abstract: Objective: to observe the influence of Danhong injection on cerebral s100 (acid calcium coupling protein) protein and NSE (neuron specificenolase) of rats undergoing cardiopulmonary bypass (CPB) and then discuss cerebral protection mechanism of the medicine. Method: 24 SD rats were divided into sham operation group, CPB group and medicine intervention group at random. Each group includes 8 rats. s100 level and NSE level of the 3 groups were compared. Results: NSE level and s100 level in the cerebral tissue homogenate of CPB group rose significantly, compared with sham operation group, $\mathrm{P}<0.01$. The difference was significant. NSE $(\mathrm{P}<0.01)$ and $\mathrm{S} 100(\mathrm{P}<0.05)$ of medicine intervention group declined significantly, compared with $\mathrm{CPB}$ group. Conclusion: Danhong injection has certain CPB cerebral protection effect.

As the most sensitive organ of human, cerebral tissue controls the whole body. Normal and stable cerebral tissue functions need relatively stable metaboilic level. Even minor injure may result in obvious structure change or function loss. In a series of bad consequences of cardiac surgery and bypass, the most common and disastrous consequence is cerebral injury[1]. To provide theoretical basis for CPB-caused cerebral injury, CPB model for rats was established, and Danhong injection was used to intervene in the model. Then, NSE level and s100 level were detected. The effect and mechanism of Danhong injection on CPB cerebral protection were discussed.

\section{Material and method}

\subsection{Material}

24 healthy and adult male clean-grade (SD) rates (provided by Animal Experiment Center of Tongji Medical College of HUST) were chosen, with the weight of (350-460)g. The animal experiment was executed in strict accordance with the experiment guide, and was approved by the Ethics Committee. The rats were divided into sham operation group, CPB group and medicine intervention group at radon. Each group includes 8 rats. 


\subsection{Method}

\subsubsection{CPB establishment}

Before the experiment, water ambrosia for $6 \mathrm{~h}$ was implemented for the rats, and $10 \%$ chloral hydrate $(300 \mathrm{mg} / \mathrm{kg}$, intraperitoneal injection) were used to narcotize the rats. The rats were fixed after oral trachea cannula with 16G trocar. Then, mechanical ventilation and oxygen intaking were conducted. The rats were monitored with electrocardiogram lead. The rats were cut longitudinally on the middle and right neck to make right carotid artery exposed. 22G trocar was used to fix the rats for CPB perfusion. Heparinization for the whole body was carried out. ACT reached (400-500)s for right atrium drainage.

CPB loop adopted right jugular vein drainage and right carotid artery perfusion. $10 \mathrm{ml}$ injector was used to drain blood by gravity at $40 \mathrm{~cm}$ below the rat's heart. CPB priming fluid consisted of hydroxyethyl starch $(12 \mathrm{ml}, 6 \%)$, sodium bicarbonate $(2 \mathrm{ml}, 5 \%)$, and mannitol $(1 \mathrm{ml})$, with the total volume of $15 \mathrm{ml}$. After fixation, vena cava drainage tube and roller blood pump were opened for bypass. When the blood in the bleeding groove became fresh red arterial blood, the blood was pumped into the right carotid artery. The initial bypass flow was $(25-35) \mathrm{ml} /(\mathrm{kg} \cdot \mathrm{min})$, and later gradually rose to $(100-150) \mathrm{ml} /(\mathrm{kg} \cdot \mathrm{min})$. After stabilization, mechanical ventilation stopped, and the oxygenator was applied. Oxygen flow and perfusion volume kept (0.8-1.0):1. After bypass for $60 \mathrm{~min}$, mechanical ventilation is restored. Then, the perfusion volume decreased gradually, and machine was stopped. In the process of CPB establishment, heart rate, mean arterial pressure and other vital sign indexes were continuously monitored.

\subsubsection{Experimental method}

Sham operation group: cannula was conducted for each part, and mechanical ventilation for 60min was implemented, without bypass.

CPB group: equivalent normal saline was added in the priming fluid, and CPB was conducted for $60 \mathrm{~min}$.

Medicine intervention group: 2ml/kg Danhong injection (manufacturer: Buchang Pharmaceuticals; batch No.: GYZZ H110247) was added in the priming fluid, and CPB was conducted for $60 \mathrm{~min}$.

\subsection{Detection index}

NST and s100 protein level in cerebral tissue homogenate were detected. After the medicine was given for $1 \mathrm{~h}, 10 \%$ chloral hydrate was used to narcotize the rats. Normal saline was used to prepare $10 \%$ cerebral tissue (left cerebrum) homogenate. The method in the specification of ELISA (enzyme linked immunosorbent assay) kit (provided by Shanghai Baoman Biotechnology Co., Ltd.) was used to determine NST and s100 protein level on enzyme-labeled instrument (US, MD Company, model: FlexStation3).

\subsection{Statistical method}

SPSS19.0 software was used to process experimental data. $\bar{x} \pm s$ was applied to describe experimental data and t test was used for contrast, $\mathrm{P}<0.05$. This means the difference has statistical significance. $\mathrm{P}<0.01$ means inter-group difference is significant. 


\section{Result}

Compared with sham operation group, NST and s100 protein level in cerebral tissue homogenate of $\mathrm{CPB}$ group rose significantly, $\mathrm{P}<0.01$. This means the difference is significant. Compared with CPB group, NSE $(\mathrm{P}<0.01)$ and S100 $(\mathrm{P}<0.05)$ level of medicine intervention group declined obviously, as shown in Tab.1.

Tab.1 ST and s100 level comparison of 3 groups

\begin{tabular}{cccc}
\hline Group & Number of samples & NSE $(\mathrm{pg} / \mathrm{mg})$ & $\mathrm{s} 100(\mathrm{pg} / \mathrm{mg})$ \\
\hline Sham operation & 8 & $46.30 \pm 5.28$ & $53.21 \pm 16.43$ \\
group & 8 & $68.28 \pm 8.80$ & $142.20 \pm 40.31$ \\
CPB group & 8 & $48.47 \pm 10.20$ & $92.10 \pm 30.01$ \\
Medicine & $/$ & -6.058 & -7.357 \\
intervention group & $/$ & -4.159 & -3.339 \\
$\mathrm{t}_{1}$ & $/$ & $<0.01$ & $<0.01$ \\
$\mathrm{t}_{2}$ & $/$ & $<0.01$ & $<0.05$ \\
$\mathrm{P}_{1}$ & & $\mathrm{P}_{2}$ &
\end{tabular}

Note: $\mathrm{t}_{1}$ and $\mathrm{P}_{1}$ represent comparison of sham operation group and CPB group; $\mathrm{t}_{2}$ and $\mathrm{P}_{2}$ represent comparison of CPB group and medicine intervention group.

\section{Discussion}

CPB cerebral protection has been the key and difficult point of medical field. CPB cerebral injury is mainly induced by CPB cerebral ischemia, cerebral anoxia, hypoperfusion state, embolism, ischemia or reperfusion injury and inflammatory reaction. If these factors further develop, neuronal death can be caused.

Danhong injection is a compound preparation extracted from salvia miltiorrhiza and safflower ${ }^{[2]}$. Compared with conventional traditional Chinese medicine, traditional Chinese medicine injection has the advantages of fast and explicit effect. Pharmacological study indicates that, Danhong injection has very effects of promoting blood circulation to remove blood stasis, and dredging the channel $^{[3]}$. It is widely applied in insufficient blood supply to the organs. In particular, it is effect for blood supply disorder of heart and head block. In recent years, many researchers have conducted large quantities of experimental and clinical researches on cerebral protection effect of Danhong injection and found that ${ }^{[4-5]}$, (1) active ingredients of tanshinone in Danhong injection can reduce the synthesis of thromboxane A2 through inhibiting PDEs (phosphodiesterase) activity, thus reducing platelet adherence and aggregation and lowering blood agglutination degree; (2) endothelium-dependent vasodilation mediated by $\mathrm{n}$ Danhong injection is endothelium-type NOS approach. hs-CRP, P-sel and ET-1 lower through synthesis of COS (cyclooxygenase)-2 and prostacyclin. Thus, vascular endothelial function improves; (3) some active ingredient in salvia miltiorrhiza are calcium ions. Through the retardant, calcium ions can expand blood vessel, relieve spasm, increase cerebral ischemia tissue engineering and improve perfusion stage; (4) Danhong injection can play certain cerebral protection role from anti-inflammation, oxidation resistance and anti-apoptosis. Meanwhile, it can obviously improve microcirculation, anti-agglomeration and antiplatelet, and reduce original level of fibrinogen.

In this research, rat CPB model was applied to further discuss cerebral protection effect of Danhong injection on CPB. The indexes chosen were NSE and s100. NSE is neuronal specific protein. Enolase widely exists in vivo and consists of $\alpha, \beta$ and $\gamma$ subunits with different immune 
properties. $\gamma \gamma$ type becomes NSE and mainly exists in neurons and neuroendocrine cells. The data indicate that, NSE level in cerebrum is the highest (accounting for about 1.55 of soluble protein in the whole cerebrum ${ }^{[6]}$ ), followed by spinal cord and peripheral nerve. In the glycolysis process, NSE can catalyze $\alpha$-phosphoglycerol to form phosphoenolpyruvic acid and play the role of maintaining normal cell metabolism. In case of neurons necrosis, NSE will leak out of cerebrum, thus leading to the improvement of NSE in cerebrospinal fluid and serum. The reason may be that neuron damage will induce metabolic disturbance of cell energy and NSE gene expression. ATP release will increase through the rise of NSE level, thus maintaining normal cell functions. Thus, NSE is used as the biochemical marker of neuron and neurogliocyte injury after CPB, and it can evaluate the cerebral injury scope and severity of illness. s100 is acid calcium coupling protein, with the molecular weight of $21 \mathrm{kD}$. It is a kind of EF cytoplasm protein ${ }^{[7]}$. s100 is a dimer consisting of two $\beta$ subunits, and is distributed at neurogliocyte of nervous system, gitter cell and anterior pituitary cerebrum. According to the data, under the normal conditions, s100 protein will not pass through blood brain barrier. If cerebral tissue is damaged, it will be expressed excessively and released into blood quickly ${ }^{[8]}$. Therefore, s100 protein can be used as the specific marker of cerebral injury.

At present, the main detection method for NSE and s100 is ELISA which has the advantages of operation simplicity, no pollution and high sensitivity. Seeing from the result, compared with sham operation group, NSE level and s100 level in CPB cerebral tissue homogenate improved significantly, $\mathrm{P}<0.01$. The difference was significant. After $\mathrm{CPB}$, cerebral injury will occur, thus leading to the rise of NSE level and s100 level. After intervention with Danhong injection, compared with CPB group, NSE level $(\mathrm{P}<0.01)$ and s100 level $(\mathrm{P}<0.05)$ in medicine intervention group reclined significantly. It thus can be seen that, Danhong injection can obviously reduce NSE level and s100 level in cerebral tissue homogenate after cerebral injury of CPB rats. This indicates that Danhong injection likely plays the cerebral protection role through down-regulating NSE and s100.

In conclusion, Danhong injection has certain CPB cerebral protection effect.

\section{References}

[1] Zhang H, Zhang J, Zha R, et al. Magnesium lithospermateB decreases [Ca2+] in endothelial cells by inhibiting $\mathrm{K}+$ currents [J]. Eur J Pharmacol, 2011, 650(1): 285-289

[2] Hang HF,Chen XQ,Hu GY,et al. Magnesium lithospermate Bdilates mesenteric arteries by activating BKCa currents and contractsarteries by inhibiting KV currents[J].Acta Pharmacol Sin '2010,31(6): 665-670.

[3] Yu He,Haitong Wan, Yueguang Du,et al. Protective effect of Danhong injection on cerebral ischemia-reperfusion injury in rats [J]. J Ethnopharmacol,2012,144(2) : 3

[4] Huang Hai-feng.Guo Fei,Cao Yuan-zhao,et al.Neuroprotec-tion by manganese supero-xide dismutase (MnSOD) mimics: an-tioxidant effect and oxidative stress regulation in acute experimen-tal stroke[J]. CNS Neurosci Therap, 2012, 10(18): 811.

[5] L Pulzova,E Bencurova, $R$ Mucha,et al. A possible role of ICAM-1 in OspA mediated borrelial adhesion to BMEC surface [J]. Int J Infect Dis, 2012,1( 16) : 237.

[6] Veeravalli K K, Rao J S. MMP-9 and uPAR regulated glioma cell migration[J].Cell Adh Migr, 2012,6( 6) : 509.

[7] Lan R,Zhang Y,Xiang J,et al. Xiao-Xu-Ming decoction pre-serves mitochondrial integrity and reduces apoptosis after focal cerebral ischemia and reperfusion via the mitochondrial p53 path-way [J]. J Ethnopharmacol, 2014, 151(1): 307.

[8] Zheng GZ, Michael C.Neuro restorative therapies for stroke: underlying mechanisms andtranslation to the clinic [J]. Lancet Neural,2009,(8):491-500. 